\title{
Battery modeling for microgrid design: a comparison between lithium-ion and lead acid technologies
}

\author{
Matteo Moncecchi, Claudio Brivio, Silvia Corigliano, Alessia Cortazzi, Marco Merlo \\ Politecnico di Milano - Department of Energy \\ Milano, Italy \\ matteo.moncecchi@polimi.it
}

\begin{abstract}
Battery energy storage systems are fundamental components in microgrids operations, therefore it is important to adopt models suitable to properly evaluate the performance of these electrical systems. Different methodologies for battery modeling have been developed and tested in this work: (i) Empirical model, in which batteries are described by analytic expressions not based on electrochemical processes; (ii) Equivalent electrical circuit model, in which batteries are described in terms of electrical quantities. These approaches allow to adapt the model to different battery technologies: both the emerging Li-ion and the consolidated lead acid are considered in this paper. The proposed models are implemented in the software Poli.NRG, a Matlab based procedure for microgrid sizing developed by Energy Department of Politecnico di Milano. Simulations are based on a real case study relevant to a microgrid in a rural area: Ngarenanyuki Secondary School in Tanzania. The proposed methodology is used to design a new microgrid based on photovoltaic and energy storage system, comparing the results obtained adopting different modeling approaches and different technologies. Eventually, results are critically analyzed and discussed in order to compare accuracy, computational effort, costs and opportunities.
\end{abstract}

Index Terms-Energy storage, Battery models, Microgrids, Rural electrification

\section{ACRONYMS}

BESS Battery Energy Storage System

SOC State of Charge

SOH State of Health

SOR State of Resistance

NPC Net Present Cost

LL Loss of Load

LLP Loss of Load Probability

LCoE Levelized Cost of Energy

OCV Open Circuit Voltage

DOD Depth of Discharge

\section{INTRODUCTION}

Distributed small scale electricity generation could result nowadays the most convenient solution to address the problem of rural electrification in developing countries, where rural areas are widespread and national grid extension costs could be prohibitive. Stand alone systems are mainly constituted of power generation units based on renewable energy sources and Battery Energy Storage System (BESS). Given the high variability and low reliability of renewable energy sources,
BESS are pivotal. Moreover, BESS represent the second major cost in stand alone systems due to their high investment cost and limited lifetime [1]. A proper battery modeling in off-grid system sizing tool is fundamental for decision makers in order to opt for the best investment. The most common battery models used in this kind of applications are the so called empirical (or analytical) models. These models represent BESS with simplified approaches, nevertheless they could introduce not negligible approximations [2]. In such a framework, the scope of the present work is to propose a novel approach to model batteries in sizing tools that can be adapted to different battery's technologies as the emerging Li-ion and the consolidated lead acid [3]. A proper battery modeling in microgrid design has to be able to estimate together the State of Charge (SOC) and the State of Health $(\mathrm{SOH})$ of the battery. The SOC is necessary to evaluate the amount of charge already stored in the battery and to compute the amount of energy that cannot be provided to the load (Loss of Load (LL)), while the SOH of the batteries takes into account bounds on lifetime due to irreversible degradation processes. Actually, battery aging can be divided into calendar and cycle aging that cause capacity and power fade. The rate of degradation depends on batteries operational and floating conditions. It is worthwhile to underline how a proper evaluation of batteries degradation is necessary to compute replacement costs.

\section{MiCROGRID SIZING METHODOLOGY CONSIDERED}

The scope of the present work is to develop a novel procedure to model battery systems in dimensioning tools for off-grid plants; in particular the proposed approaches are integrated in the tool Poli.NRG, a new methodology developed by the Energy Department of Politecnico di Milano for sizing stand alone systems. Poli.NRG is a comprehensive procedure, written in Matlab environment, that allows for a robust design of a photovoltaic (PV)+BESS plant: it couples the atypical features of rural contexts (i.e. unpredictability of energy sources and load consumption uncertainties) with proper component models, by including estimation errors into the design phase [4]. The software is composed of four building blocks, related to different phases of the design procedure. 
1) Data inputs gathering: information regarding electricity consumption, power generation, fixed and variable equipment costs and weather data are collected.

2) Inputs processing: the collected input data are processed in order to obtain daily load profiles and power generation profiles. Load profiles are generated with the subroutine LoadProGen which, using a stochastic approach, is able to formulate different daily load profiles starting from field data [5]. Yearly profile is generated by aggregating randomly the daily load profiles and lifetime load profiles are obtained by assuming possible load evolution scenarios.

3) System modeling and simulation: this block includes mathematical models of system's components, namely $\mathrm{PV}$ and BESS. Simulations run with a time step $\Delta k$ of one minute during all the plant lifetime $L T$. At each time step $k$, the energy required or provided to the batteries is given by an energy balance between load consumption and photovoltaic power generation:

$$
E_{\text {batt }}(k)=E_{P V}(k)-\frac{E_{\text {load }}(k)}{\eta_{\text {inv }}}
$$

Where $E_{\text {load }}(k)$ is the load consumption, $E_{P V}(k)$ is the production from $\mathrm{PV}$ and $\eta_{i n v}$ is the inverter efficiency. Microgrid optimal design is based on performance indexes: Loss of Load Probability (LLP) is the amount of energy not provided to the load (LL) over the total energy required during the plant lifetime.

$$
L L P=\frac{\sum_{k=1}^{L T} L L(k)}{\sum_{k=1}^{L T} E_{l o a d}(k)}
$$

Net Present Cost (NPC) is the present value of all the costs of investment (Inv) and operation of the system $(O \& M)$ over the project lifetime, it is evaluated as the sum of the net cash flows during each year $y$ actualized with the expected discount factor $r$.

$$
N P C=\sum_{y=1}^{L T} \frac{\operatorname{Inv}(y)+O \& M(y)}{(1+r)^{y}}
$$

Levelized Cost of Energy (LCoE) is an indicator that measures lifetime costs divided by energy production.

$$
L C o E=\frac{r(1+r)^{L T}}{(1+r)^{L T}-1} \frac{N P C}{(1-L L P) \sum_{k=1}^{L T} E_{l o a d}(k)}
$$

4) Output formulation: a heuristic optimization method is used to find the most robust design of the systems. The optimization algorithm is divided in two steps: firstly, the searching space is defined, i.e. the ranges of PV and BESS to be investigated; secondly, the optimal combination of PV and BESS is found within the searching space (for more details about the equations and the architecture of the adopted procedure see [4] and [6]). The second step utilizes a heuristic procedure to find the optimal plant size: the adopted algorithm is based on the imperialistic competitive algorithm that is an iterative process progressively exploring the searching space. The optimum solution is found as the combination of PV+BESS having the minimum NPC whilst respecting LLP constraint of 5\%. The new load profiles are simulated until a convergence criterion is fulfilled.

The tool is available free of charge [7].

\section{BESS MODELS INVESTIGATED}

Three different models of BESS have been developed and integrated in the tool Poli.NRG. These models are detailed in the following paragraphs.

\section{A. M1 - Simplified empirical model}

It is the simplest, but also the most common, model found in scientific literature [8]. Battery is characterized by constant efficiency that takes into account energy losses during charge $\left(\eta_{c h}\right)$ and discharge $\left(\eta_{\text {dis }}\right)$. The energy flows entering $E_{c h}(k)$ or exiting $E_{d i s}(k)$ the battery at each time step $k$ are computed as follows:

$$
\begin{gathered}
E_{c h}(k)=E_{\text {batt }}(k) \cdot \eta_{c h} \\
E_{\text {dis }}(k)=\frac{E_{\text {batt }}(k)}{\eta_{\text {dis }}}
\end{gathered}
$$

where $E_{\text {batt }}(k)$ is defined in eq. 1 . The SOC of the battery at each time step is computed as:

$$
S O C(k)=S O C(k-1)+\frac{E_{c h / d i s}(k)}{E_{B E S S}}
$$

where $E_{B E S S}$ is the nominal capacity of the battery. The model is subject to performance constraints:

- Maximum value for the power to energy ratio $P E_{\text {ratio }}$, which is the maximum power input/output with respect to the rated capacity of the batteries. Given a time step $\Delta k$, the maximum energy the battery can provide $E_{\max }$ is defined as:

$$
E_{\text {max }}=E_{B E S S} \cdot P E_{\text {ratio }} \cdot \Delta k
$$

If the battery is asked to provide an energy $E_{b a t t}(k)$ greater than $E_{\max }$, a loss of load has to be taken into account:

$$
L L(k)=\left(\left|E_{\text {batt }}(k)\right|-E_{\text {max }}\right) \cdot \eta_{\text {inv }}
$$

- Minimum and maximum value for SOC, that will prevent permanent damage to the battery. As a consequence, if the battery is asked to discharge to a $\operatorname{SOC}^{t h}(k)$ that is lower than the minimum SOC admissible $S O C_{m i n}$, the state of charge at time step $k$ is saturated to $S O C_{\text {min }}$ and the energy required by the loads that the system is not able to supply is evaluated as LL:

$$
L L(k)=\left(S O C_{m i n}-S O C^{t h}(k)\right) \cdot E_{B E S S} \cdot \eta_{d i s} \cdot \eta_{i n v}
$$

The $\mathrm{SOH}$ is not estimated by the model. To account for degradation, some constraints are imposed to the battery: 
- Maximum number of cycles before replacement, to account for cycle aging. The equivalent cycle $\left(E q_{\text {cycle }}\right)$ linked to the single time step is calculated as:

$$
E q_{\text {cycle }}(k)=\left|\frac{S O C(k)-S O C(k-1)}{2}\right|
$$

Then, the amount of equivalent cycles $\left(E q_{\text {cycles }}\right)$ are computed as follows:

$$
E q_{\text {cycles }}(k)=E q_{\text {cycles }}(k-1)+E q_{\text {cycle }}(k)
$$

- Maximum number of years before replacement, to account for calendar aging.

\section{B. M2 - Complete empirical model}

It is an improved class of the model previously described. Given the evidence that battery efficiency depends on E-rate [9], which is defined as the ratio of power to rated energy:

$$
E_{\text {rate }}(k)=\frac{\left|P_{\text {batt }}(k)\right|}{E_{B E S S}}=\frac{\left|E_{\text {batt }}(k)\right| / \Delta k}{E_{B E S S}}
$$

a cubic correlation has been adopted to properly model efficiency degradation at higher E-rate.

$$
\eta(k)=a E_{\text {rate }}^{3}+b E_{\text {rate }}^{2}+c E_{\text {rate }}+d
$$

Similarly, cycle aging is taken into account with a decrease of $\mathrm{SOH}$ during lifetime that is proportional to the number of cycles and to the capacity fade index $c f$

$$
S O H(k)=S O H(k-1)-E q_{c y c l e}(k) \cdot c f(k)
$$

$c f$ differs according to the technology: is a function of Erate when considering lithium ion batteries and a function of the depth of discharge (DOD) when considering lead acid batteries [10] [11].

Thereafter, in the proposed model the constraints that limit battery lifetime are:

- Minimum SOH before replacement, to account for cycle aging;

- Maximum number of years before replacement, to account for calendar aging.

In this model, SOC at each time step is properly computed taking into account capacity fade:

$$
S O C(k)=S O C(k-1)+\frac{E_{c h(k) / d i s(k)}}{E_{B E S S} \cdot S O H(k)}
$$

\section{M3 - Electrical model}

In the electrical models, batteries are represented by equivalent electrical circuits that aim to model as accurately as possible operation parameters, e.g. voltage and current characteristics at the external terminals. Models can be defined with respect to time or frequency domain according to the deployed circuital elements [12] [13]. A simplified electrical equivalent circuit model is proposed as a valid alternative to the more widespread analytic models. The proposed circuit (Fig. 1) is a passive model. It is composed of a capacitance $C_{b}$ that represents battery equilibrium condition, in series with a resistance $R_{\text {int }}$ to account for losses during operation; both the parameters depend on SOC and aging conditions. Voltage across $C_{b}$ is the Open Circuit Voltage (OCV) while voltage drop across $R_{\text {int }}$ is the overpotential of the cell. The approach proposed to set the relation between internal resistance and SOC is based on electrochemical impedance spectroscopy: laboratory testing has to be performed at different battery SOC to define the resistance as the real part of impedance at a specific frequency [14]. Reference frequency is related to the load input profile; while $C_{b}$, as a function of OCV (Fig. 2), is determined from the cell discharge curve (Fig. 3) by applying the capacitor's constituent equation.

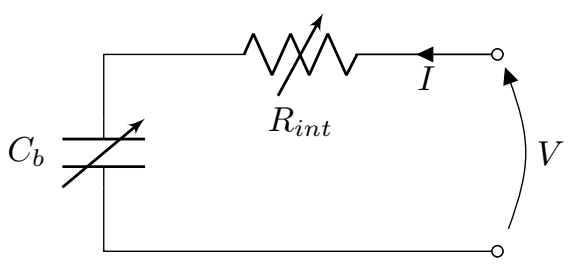

Fig. 1. Electrical circuit model for BESS.

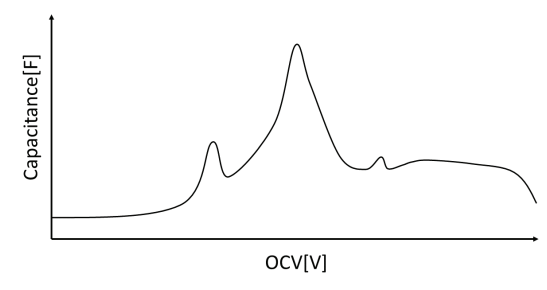

Fig. 2. Intercalation capacitance vs OCV.

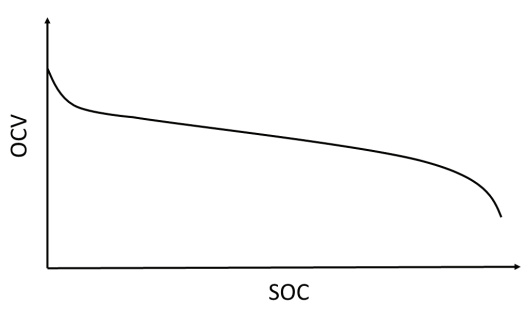

Fig. 3. Open circuit voltage vs SOC.

The following equations characterize the cell:

$$
\left\{\begin{array}{l}
I=P_{\text {cell }} / V \\
I=C_{b}(O V C) \cdot \frac{d O V C}{d t} \\
V=O C V(S O C)+R_{\text {int }}(S O C) \cdot I
\end{array}\right.
$$

In order to be implemented in Poli.NRG, they have to be solved numerically. The power flowing in each cell is defined as:

$$
P_{\text {cell }}(k)=\frac{E_{\text {batt }}(k)}{\Delta k \cdot N_{\text {cells }}}
$$

The number of cells $N_{\text {cells }}$ is equal to the size of the BESS divided by the capacity of the cell. Therefore, the current $I(k)$ can be computed as:

$$
I(k)=\frac{P_{c e l l}(k)}{V(k-1)}
$$


OCV $(\mathrm{k})$ and $\mathrm{V}(\mathrm{k})$ are updated accounting for $C_{b}$ and $R_{\text {int }}$ at the previous time step:

$$
\begin{aligned}
& O C V(k)=O C V(k-1)+\frac{I(k)}{C_{b}(k, O C V(k-1))} \\
& V(k)=O C V(k)+R_{\text {int }}(k, S O C(k-1)) \cdot I(k)
\end{aligned}
$$

The constraints that limit the flows through the battery are:

- Maximum power to energy ratio $P E_{\text {ratio. }}$ In the case in which $E_{\text {batt }}(k) \geq E_{\max }$ the BESS is not able to provide all the energy required to feed the loads. The loss of load of the system is computed as:

$$
L L(k)=\left(\left|E_{\text {batt }}(k)\right|-E_{\max }\right) \cdot \eta_{\text {inv }}
$$

- Maximum and minimum voltage. Voltage limits are correlated to battery SOC and to the charging/discharging current; when the theoretical voltage $V^{t h}(k)$ that the battery should reach at time step $k$ is lower than $V_{\text {min }}$, the battery is not able to feed the load and a loss of load is computed as:

$$
L L(k)=\left|E_{\text {batt }}(k)\right| \cdot \eta_{\text {inv }}
$$

Cycle aging is taken into account with SOH and SOR (State of Resistance) indicators. SOH variation during lifetime is the same as for empirical model; SOR accounts for resistance growth due to degradation process inside the battery. It increases with the number of cycles for lead acid cell and with the number of cycles and C-rate in lithium ion.

The capacity $C_{b}$ and the resistance $R_{\text {int }}$ are updated among the simulations to take into account degradation:

$$
\begin{gathered}
C_{b}(k)=C_{b}(0) \cdot S O H(k) \\
R_{\text {int }}(k)=R_{\text {int }}(0) \cdot \operatorname{SOR}(k)
\end{gathered}
$$

Battery is replaced when either $\mathrm{SOH}$ has reached the minimum value (cycle aging) or the battery has reached the maximum number of years (calendar aging).

The three models have been parametrized for a lithium and lead acid cell with data taken from literature, datasheets and experimental measurements. The electrical model for a lithium ion cell has been validated with measures taken at the Energy Storage Research Center (ESReC) located in Nidau $(\mathrm{CH})$. The measurements were carried out within the framework of the collaboration between Politecnico di Milano (DoE) and CSEM-PV Center (Swiss Center for Electronics and Microtechnology). The tested cell is the Lithium Nickel Cobalt Oxide (LNCO) cell BostonPower Swing5300 [15]. Detailed description about laboratory testing is reported in [14]. It is worthwhile to depict that the proposed models are capable to represent many different energy storage technologies; in order to demonstrate the effectiveness of the approach, some numerical simulation have been performed on the aforementioned commercial product.

\section{Simulation AND RESUlts}

A real life case study has been taken into consideration: a microgrid feeding the Ngarenanyuki Secondary School, in Tanzania [16]. The energy consumption of the school has been monitored since 2015. Through a field survey, data about the loads have been collected and consumption has been classified in daily loads (e.g classroom lights, household appliances, water pump) and occasional three phase loads (mainly a mill machine). They were used to generate stochastic load profiles extended for the entire plant lifetime (20 years) considering a constant yearly energy consumption. Cost figure about PV modules, batteries and off-grid inverters are collected from a survey among Tanzanian local suppliers, while O\&M, other investment costs and modeling parameters have been estimated based on experience. Eventually, the procedure has been executed over the system lifetime to size a new microgrid based on PV and BESS, devoted to substitute the already present undersized stand alone system. In particular, the three proposed BESS models have been implemented with the two driving technologies Li-ion and lead acid and eventually they have been compared in terms of sizing results, costs and simulation time. Tables I and II summarize the BESS parameters in input to Poli.NRG.

TABLE I

LITHIUM ION BATTERY SPECIFICATIONS

\begin{tabular}{|l|c|c|c|}
\hline \multirow{2}{*}{} & \multicolumn{3}{|c|}{ Lithium ion } \\
\cline { 2 - 4 } & M1 & M2 & M3 \\
\hline SOC initial & 1 & 1 & 1 \\
PEC min & 0 & 0 & - \\
SOH min [kW/kWh] & 2 & 2 & 2 \\
Calendar life [years] & - & 0.8 & 0.8 \\
Cycle life [n cycles] & 2000 & 12 & 10 \\
Charge efficiency \% & 97.5 & $\mathrm{f}\left(E_{\text {rate }}\right)$ & $\mathrm{f}\left(C_{\text {rate }}\right)$ \\
Discharge efficiency \% & 97.5 & $\mathrm{f}\left(E_{\text {rate }}\right)$ & - \\
Cell capacity [Ah] & - & - & 5.3 \\
Max voltage [V] & - & - & 4.2 \\
Min voltage [V] & - & - & 2.75 \\
\hline
\end{tabular}

TABLE II

LEAD ACID BATTERY SPECIFICATIONS

\begin{tabular}{|l|c|c|c|}
\hline \multirow{2}{*}{} & \multicolumn{3}{|c|}{ Lead Acid } \\
\cline { 2 - 4 } & M1 & M2 & M3 \\
\hline SOC initial & 1 & 1 & 1 \\
SOC min & 0.5 & 0.5 & - \\
PE ratio [kW/kWh] & 0.25 & 0.25 & 0.25 \\
SOH min & - & 0.8 & 0.8 \\
Calendar life [years] & 8 & 8 & 8 \\
Cycle life [n cycles] & 1500 & f(DOD) & f(DOD) \\
Charge efficiency \% & 90 & $\mathrm{f}\left(E_{\text {rate }}\right)$ & - \\
Discharge efficiency \% & 90 & $\mathrm{f}\left(E_{\text {rate }}\right)$ & - \\
Cell capacity [Ah] & - & - & 10 \\
Max voltage [V] & - & - & 2.17 \\
Min voltage [V] & - & - & 2 \\
\hline
\end{tabular}

\section{A. Operation simulation}

Poli.NRG simulates the operation of the PV+BESS system for the whole lifetime of the microgrid and it evaluates for 
each time step $k$ the values of the variables of the system. The proposed approach is based on simulation detailed in time step limited to one single minute. This is to properly evaluate fluctuations in load/generation power profiles. Consequently BESS injections/withdrawals are solved each minute driving to an accurate evaluation of the BESS performances and bounds. As an example, when considering complete empirical model M2 the efficiency of the BESS changes according to the state of charge and the charging/discharging power at every single time step. In Fig. 4 an example of the efficiency for the optimal size of BESS is plotted both for Li-ion system and for lead acid. Similarly, the proposed approach allows a clear evaluation of the equivalent cycles dispensed by the BESS (Fig. 5). Equivalent cycles affect the performance of the BESS and the number of times it has to be changed over the total lifetime of the microgrid.

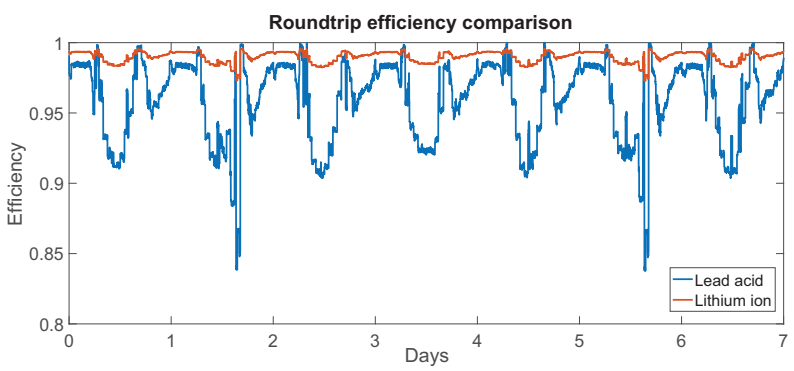

Fig. 4. Comparison of the efficiency trend for Li-ion and lead acid (optimal size of BESS simulated for seven days).

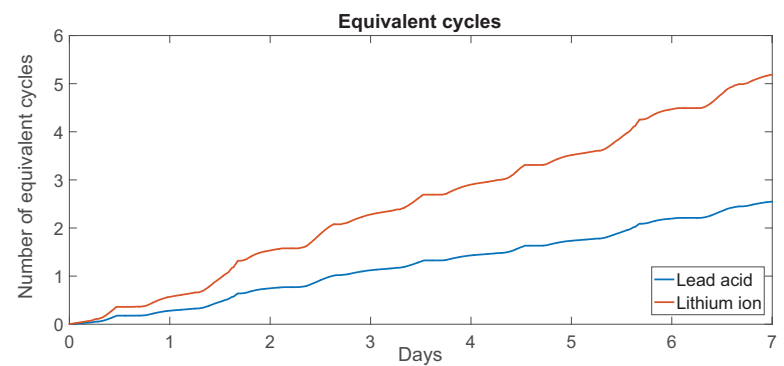

Fig. 5. Comparison of the equivalent cycles for Li-ion and lead acid (optimal size of BESS simulated for seven days).

\section{B. Robust design}

Figures 6 and 7 display the maps of solutions of the simulations with the three BESS models proposed for lead acid and lithium ion technology. The map of solutions is composed of different areas of solutions that are related to the specific BESS model adopted in the simulation. Each area of solutions represents with contour lines the resulting optimal combinations of PV and BESS size among the simulated lifetime profiles LC. The contour line of the optimal combinations is a curve along which specific combinations have appeared with the same frequency (i.e., it represents isolines). The robust design (BESS robust and $P V_{\text {robust }}$ ) is evaluated as the combination that resulted to be the optimal with the highest frequency (Table III). NPC, LCOE and LLP are evaluated as the mean values of each solution in which $B E S S_{\text {robust }}$ and $P V_{\text {robust }}$ appear. The parameter $L C_{\text {robust }}$ represents the number of simulated load profiles until convergence. Every simulation provides an optimal solution, since the dispersion of the optimal solutions is limited, the convergence criterium is respected and the sizing with the high frequency of occurrence is selected as the global optimum of the robust design process.

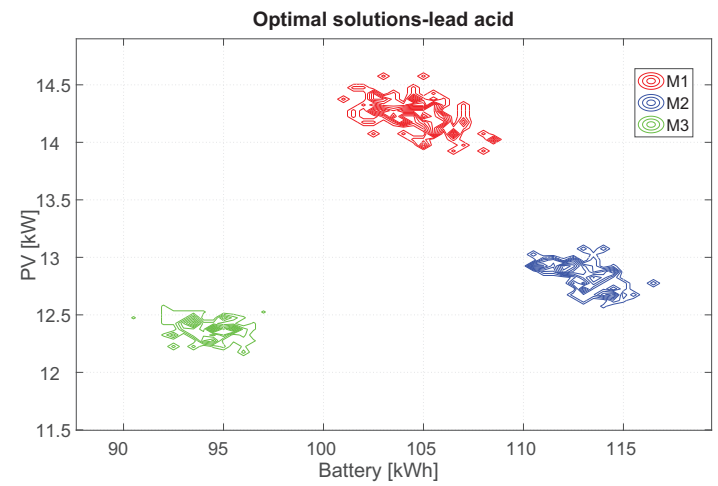

Fig. 6. Sizing results of Poli.NRG with lead acid BESS systems.

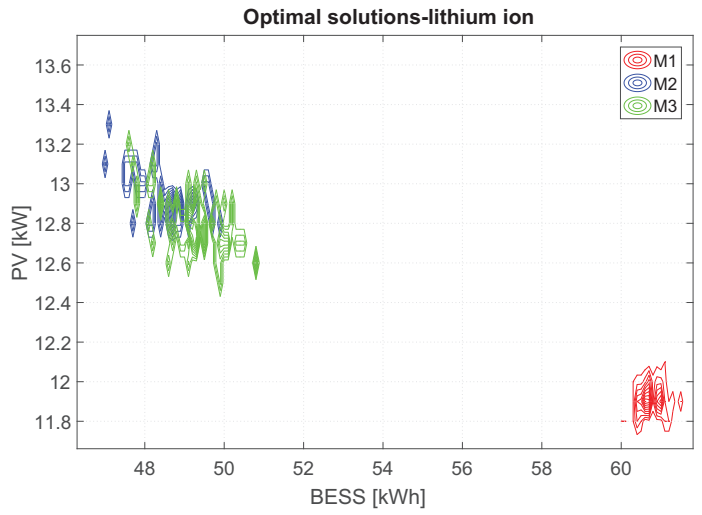

Fig. 7. Sizing results of Poli.NRG with Li-ion BESS systems.

\section{Lead acid versus Li-ion technology}

Regarding lead acid technology, the empirical model tends to overestimate system size with respect to the robust design using electrical model $(+18 \%)$, as it can be seen from Table III. The overestimation can be caused both by the different estimation of SOC and SOH and by the different parametrization of Model 2 and Model 3. For the simulations, Model 2 is based on manufacturer's data, while Model 3 relies on experimental data. This could motivate the gap between the NPC estimations. In the simulation performed, BESS optimal size calculated adopting the simplified empirical model (M1) is an intermediate value between the other two models while PV size is noticeably bigger than $P V_{\text {robust }}$ provided by M2, due to the fact that battery operates with a lower efficiency in M1 with respect to M2. This implies that even if the parameters in input to the simplified empirical model (efficiency and 
TABLE III

RESULTS OF POLI.NRG SIMULATIONS WITH DIFFERENT BATTERY TECHNOLOGIES AND MODELING APPROACHES

\begin{tabular}{|c|c|c|c|c|c|c|c|c|}
\hline $\begin{array}{c}\text { BESS } \\
\text { technology }\end{array}$ & $\begin{array}{c}\text { BESS } \\
\text { model }\end{array}$ & $\begin{array}{c}P V_{\text {robust }} \\
{[k W]}\end{array}$ & $\begin{array}{c}B E S S_{\text {robust }} \\
{[k W h]}\end{array}$ & $\begin{array}{c}\text { NPC } \\
{[k \$]}\end{array}$ & $\begin{array}{c}\text { LCoE } \\
{[k \$ / k W h]}\end{array}$ & $\begin{array}{c}\text { LLP } \\
{[\%]}\end{array}$ & $\begin{array}{c}\text { Simulation } \\
\text { time }[\mathrm{min}]\end{array}$ & $\begin{array}{c}\text { LC } C_{\text {robust }} \\
{[\#]}\end{array}$ \\
\hline \multirow{3}{*}{ Lead acid } & M1 & 14.2 & 105 & 119.82 & 0.644 & 4.99 & 8 & 114 \\
& M2 & 12.9 & 112 & 118.66 & 0.637 & 4.99 & 45 & 124 \\
& M3 & 12.35 & 94.5 & 114.85 & 0.617 & 4.99 & 67 & 114 \\
\hline \multirow{3}{*}{ Li-ion } & M1 & 11.9 & 60.6 & 120.50 & 0.648 & 4.90 & 7 & 101 \\
& M2 & 12.9 & 48.6 & 116.28 & 0.625 & 4.99 & 45 & 108 \\
& M3 & 12.8 & 49.1 & 116.22 & 0.624 & 5 & 140 & 98 \\
\hline
\end{tabular}

maximum number of cycles) are correctly estimated, simulation results could not resemble the ones of more sophisticated approaches. Considering Li-ion technology simulations, electrical and empirical models result to have very similar system sizes while the simplified empirical model shows a non negligible difference. The resulting NPC is very similar between M3 and M2 methodologies while it is significantly higher when using the more simplified approach. The results' similarity among M2 and M3 for lithium ion is likely due to models' parametrization: the values of the variable parameters of the different BESS models and degradation curves have been computed from the same experimental measurements.

\section{Computational time}

As reported in Table III, computational time for simulating one lifetime load profile LC strongly varies with the BESS modeling approach adopted (to the present work, an Intel i7 $4700 \mathrm{k}-16 \mathrm{~Gb}$ has been used). Empirical model takes almost 6 times the simulation time needed for simplified empirical model, both for lead acid and lithium ion technologies; electrical lead acid model computational effort is 8 times higher than the simplest model, while for lithium ion batteries is even 18 times higher.

\section{E. Cost analysis}

In the study performed lead acid batteries have been assumed at $150 \$ / \mathrm{kWh}$, while Li-ion option at $400 \$ / \mathrm{kWh}$. Nevertheless, for a proper economics evaluation, it is necessary to account also for batteries' replacement costs for a proper analysis of the costs associated to each technology. Actually, lead acid batteries have to be replaced three times during plant lifetime while lithium ion batteries only one. For this reason NPC of the plant with lithium ion batteries is only 2000 \$ higher than with lead. Moreover, the LCOE of the two technological options are considerably similar.

\section{CONCLUSions}

Three modeling approaches have been proposed and parametrized both for lithium and lead acid technologies. The models differ greatly in terms of computational effort: the higher the model's complexity, the higher the computational time. The empirical simplified model M1 leads to an oversizing of the plant with consequent overestimation of investment costs. Complete empirical (M2) and electrical models (M3) are instead comparable when data are taken from laboratory measurements. In conclusion it is relevant to highlight the necessity of a proper and smart BESS modeling when facing off-grid systems design. The models proposed have been coded in a tool (free of use) designed to support investors in the choice of the most robust solution for the study case under investigation.

\section{ACKNOWLEDGMENT}

Authors thank Dr. Vincenzo Musolino of the PV-Center CSEM SA for the support in the development of the electrical battery model.

\section{REFERENCES}

[1] International Renewable Energy Agency, "Solar Pv in Africa: Costs and Markets", September 2016.

[2] Geoffrey T. Klise and Joshua S. Stein, "Models Used to Assess the Performance of Photovoltaic Systems", 2009.

[3] International Renewable Energy Agency, "Electricity storage and renewables: Costs and markets to 2030", October 2017.

[4] Claudio Brivio, Matteo Moncecchi, Stefano Mandelli and Marco Merlo, "A novel software package for the robust design of off-grid power systems", Journal of Cleaner Production, 166: pp.668-679, 2017.

[5] Stefano Mandelli, Marco Merlo and Emanuela Colombo, "Novel procedure to formulate load profiles for off-grid rural areas", Energy for Sustainable Development, 31:130-142, April 2016.

[6] Stefano Mandelli, Claudio Brivio, Emanuela Colombo and Marco Merlo, "A sizing methodology based on Levelized Cost of Supplied and Lost Energy for off-grid rural electrification systems", Renewable Energy, 89: pp.475488, 2016.

[7] www.facebook.com/energy4growing2014/

[8] Nicholas Etherden and Math H. J. Bollen, "Dimensioning of energy storage for increased integration of wind power", IEEE Transactions on Sustainable Energy, 4(3): pp.546-553, 2013.

[9] Massoud Pedram and Qing Wu, "Battery-powered digital CMOS design", Proceedings - Design, Automation and Test in Europe, DATE, 10(5): pp.72-76, 1999.

[10] S. M. G. Mousavi and M Nikdel, "Various battery models for various simulation studies and applications. Renewable and Sustainable Energy Reviews", 32:477-485, 2014.

[11] Lijun Gao, Shengyi Liu and Roger A Dougal, Dynamic Lithium-Ion Battery Model for System Simulation. IEEE Transactions on Components and Packaging Technologies, 25(3):495-505, 2002.

[12] V Johnson. Battery performance models in ADVISOR. Journal of Power Sources, 110(2):321-329, 2002.

[13] Luiz Carlos Stevanatto, Valner Joao Brusamarello, and Stanislav Tairov. Parameter identification and analysis of uncertainties in measurements of lead-acid batteries. IEEE Transactions on Instrumentation and Measurement, 63(4):761-768, 2014.

[14] Claudio Brivio, "Battery Energy Storage Systems: Modelling, Applications and Design Criteria", PhD thesis, Politecnico di Milano, 2017.

[15] Datasheet Boston Power Swing 5300, http://www.boston-power.com.cn

[16] Stefano Mandelli, Claudio Brivio, Matteo Leonardi, Emanuela Colombo, Marta Molinas, Eugene Parkc and Marco Merlo, "The role of electrical energy storage in sub-Saharan Africa", Journal of Energy Storage, Volume 8, 2016, Pages 287-299, 